\title{
Hohle Gasse
}

\section{Frank Fischer}

Korrespondenz:

Frank Fischer

Facharzt Psychiatrie und Psychotherapie Pfruendhofstrasse 54 CH-8910 Affoltern
A. trug die gleiche Trainerhose wie an unserem allerersten Gespräch, nur der Riss an der Seite war neu. Er starrte nach unten. Am linken Auge zuckte ein Muskel.

«Was soll ich machen?», fragte er.

Ich zuckte auch, aber mit den Schultern. Ich wusste nicht mal, von was er jetzt lebte. Zur Sozialhilfe jedenfalls ging er nicht. Damit hätte er die Schlussrunde eingeleitet. Die Fremdenpolizei fragte dort vor jeder Passverlängerung, ob er Fürsorgegelder bezog. Seine Aufenthaltsbewilligung war an Arbeit geknüpft und wurde nur verlängert, wenn er Arbeit hatte. Oder sich mindestens selber unterhalten konnte. Der Gang zur Sozialhilfe war also heikel. Da spielte es auch keine Rolle, dass er seit 15 Jahren in der Schweiz lebte.

A. war krank und arbeitsunfähig, das konnte ich ihm bescheinigen. Aber die Invalidenversicherung anerkannte keine normalen Arztzeugnisse mehr. Bei über 1 Milliarde Franken Defizit pro Jahr und inzwischen 7 Milliarden Franken Schulden hatte sie angefangen, auf Zeit zu spielen. Viel zu wenig Profis für die wachsende Zahl der Dossiers. Den Erfolg, dank verschleppter Verfahren, las man bereits in der Presse: deutlicher Rentenrückgang im laufenden Halbjahr.

A. hatte Anspruch auf berufliche Wiedereingliederung und, wenn die nicht möglich war, auf Rente. Theoretisch. Die Versicherung lehnte beides ab. Die Gründe waren dünn und würden vor Gericht nicht ausreichen. Aber jeder Instanzschritt davor dauerte inzwischen über ein Jahr. A. hatte weder das Geld für den Anwalt noch die Zeit zu warten - und einen abgelaufenen Ausweis. Der nicht verlängert wurde. Recht haben und Recht bekommen, das war zweierlei.

Also doch arbeiten, um zu bleiben? Was kümmert die Gesundheit, wenn es ums Überleben geht? Der letzte Arbeitsversuch im Restaurant eines ehemaligen Kollegen ging allerdings keinen Monat gut. So depressiv wie A. um die Tische zitterte, brauchte er als Kellner nicht mehr anzutreten. Der Kollege nahm ihn daraufhin nach hinten, in die Küche. Aber hier war A. zu langsam, ausserdem gab es zu wenig Arbeit.

Für den Bau oder die Fabrik taugte er wegen seiner Schmerzen erst recht nicht mehr. Nicht mal Autofahren konnte er längere Strecken.
Alle zwei Wochen rief ich im Rechtsdienst der Invalidenversicherung an. Der Anwalt stellte einen baldigen Entscheid in Aussicht. Alle zwei Wochen neu. Ich wies auf die Dringlichkeit hin, der Entscheid kommt, sagte der Anwalt. Alle zwei Wochen neu. Ich erwähnte den gescheiterten Arbeitsversuch, die verschlechterte Gesundheit, der Anwalt forderte ein aktuelles Zeugnis an, mit ausführlichem Bericht. Dann werde der Entscheid kommen.

Ich versuchte etwas, das ich noch nie in einem Therapiegespräch gemacht hatte. Könnten Sie nicht Autos verschieben? Oder andere Waren? Und zu Hause verkaufen. Oder umgekehrt. Von mir aus illegal.

Wie meinen Sie das?

Keine Ahnung. Aber zwischen Ausweisung und Suizid musste es verdammt noch mal doch noch mehr Möglichkeiten geben.

Um die Kündigung der Wohnung abzuwenden, hatte er einen Kredit aufgenommen. Jemand in Luzern, aus welcher Szene auch immer, lieh ihm 2000 Franken. Für 4 Monate. Und für 400 Franken Zins pro Monat.

Warum haben Sie das gemacht? Das ist doch Wucher, hatte ich gefragt.

Hätten Sie mir das Geld geliehen?, fragte er zurück.

Sicher nicht. Das Geld war weg. Der Hai in Luzern glaubte vielleicht an seine eigenen Methoden, um sich sein Geld zurückzuholen. Aber das war rechtsfreier Raum. Und für A. ging es eh um alles. Zeit schinden, bis vielleicht doch ein Entscheid der Versicherung kam. Und dann könnte er auf die Gemeinde oder von der Rente zurückzahlen. Und wenn kein Entscheid kam, würde er sowieso untertauchen müssen.

$\mathrm{Zu}$ meinem Glück rauchte A., vor und nach dem Gespräch, draussen, am Fenster der Sekretärin. So seltsam das klingt, aber es beruhigte mein Gewissen. So gross war die Geldnot also doch nicht.

Was soll ich tun, hatte er gefragt. Zum Beispiel zur Familie zurückkehren. Er war seit Jahren nicht mehr zu Hause gewesen und hatte noch länger kein Geld mehr geschickt. Wahrscheinlich waren die aber froh, wenn sie ihn nicht mehr sahen. Mindestens nicht krank und mittellos. 
Die wenigen Sätze, die er über seine Frau und die beiden erwachsenen Kinder zu Hause verloren hatte, liessen darauf schliessen.

Ich hatte angefangen, darüber nachzudenken, ob ich ihn nicht beschäftigen könnte. Auf privater Basis. Ihn zum Beispiel etwas aufbauen lassen, in seinem zerstörten Heimatdorf. Eine Werkstatt. Die er nur beaufsichtigen müsste. Und einen Austausch von Lehrlingen hier und dort organisieren. Aber wahrscheinlich überschätzte ich ihn. Und meine Möglichkeiten. Er war auch nicht der einzige mit richtigen Problemen. Und zum Glück war er nicht sympathisch. Es blieb bei meinen Gedankenspielen.
Mir viel nichts mehr ein. Über Medikamente mussten wir nicht mehr reden. Er schluckte die ganze Palette. Aber der Gegenwert in Geld hätte ihm wahrscheinlich mehr genutzt. Ich gab ihm einen neuen Termin.

Zwei Monate später kam der ablehnende Entscheid der Invalidenversicherung. Ich machte Einsprache für ihn und wir warteten. Der Bescheid der Fremdenpolizei kam wenige Tage später per Einschreiben, eine Frist von 4 Wochen war gesetzt. $\mathrm{Zu}$ einem Gespräch kam A. noch, beim Folgetermin, kurz vor Ablauf der Frist, blieb er aus. Ich habe nie wieder von ihm gehört.

\section{Prinzessin}

\section{Peter Süsstrunk}

Korrespondenz:

Peter Süsstrunk
Die zierliche, wenn auch nicht magersüchtige Prinzessin B. hatte zwar, als sie noch Teenager gewesen war, von Journalisten den inoffiziellen Übernamen «die Gazelle» erhalten, entwickelte sich jetzt aber zunehmend zu etwas, das eher die Bezeichnung «die Zicke» verdiente. Einen wertvollen Fingerring, den sie grossmütterlicherseits geerbt hatte und der ihr allerdings zu gross war, schmiss sie kurzerhand in den Froschteich, welcher sie auch noch auf die Idee brachte, in die Schlossküche zu laufen, um das Personal zu bezichtigen, der königlichen Familie regelmässig einen regelrechten Schlangenfrass vorzusetzen: Statt dessen wünsche sie, nämlich Prinzessin B., jetzt Delikatessen, z.B. Froschschenkel!

Der Küchenchef, schwer beleidigt und den Tränen nahe, beklagte sich beim König, der sich über das allürenhafte Benehmen seiner Tochter ohnehin bereits Sorgen machte und sie zu einer Audienz bestellte. Als sie voll Trotz und felsenfest entschlossen, sich von ihrem Vater nichts sagen zu lassen, erschien, beschränkte er sich allerdings darauf, sie lange schweigend anzusehen, nicht ohne anschliessend dem Küchenchef auszurichten, für die königliche Familie genügten weiterhin einfache Mahlzeiten, wozu etwas wie Froschschenkel weiterhin nicht gehöre.

Prinzessin B. liess ihren Ärger darüber handkehrum an ihren ziemlich zahlreichen Freiern aus, denen sie Vorträge hielt: Wieso sie denn ausgerechnet eine Königstochter zu ehelichen wünschten, wenn doch einfache Girls aus dem einfachen Volk vollends genügen würden und man(n) nicht über dem Stand heiraten sollte? Wieso über dem Stand?, verteidigte sich ein aus angeblich fürstlicher Familie stammender Freier. Das eben sei das Problem, so die Antwort der Prinzessin: Da stehe er, nämlich der Kandidat, wie ein Pfau vor ihr und schlage sein Rad, mache sich gross, erwähne beiläufig seine Tüchtigkeit sowie Zuverlässigkeit, und das Ganze mit geschwellter Brust, als wäre er der Himmel oder das Schicksal persönlich; es wundere sie nur, dass er nicht noch nach einer Gelegenheit suche, sein piekfeines Jackett auszuziehen, um seine allenfalls nicht unansehnlichen Muskeln unterm Hemd vorzuführen!

Es sei doch von der Natur nicht anders gewollt, versuchte, bereits leicht verlegen, der junge Fürst sich zu wehren - dass sich nämlich der Kandidat von seiner besten Seite und möglichst imstand zeige, seiner Angebeteten wenigstens die Illusion zu vermitteln, es handle sich bei ihm um den Idealfall eines wünschenswerten Ehepartners, als den sie ihn wenigstens in Betracht ziehen dürfe.

Darauf ging die Prinzessin keineswegs ein: Wenn sich die Natur, um zu ihrem Recht zu gelangen, der Illusion bedienen müsse, dürfe an dieser Methode wenigstens leicht gezweifelt werden; ja, die Natur selbst erlaube sich vielleicht, das weibliche, üblicherweise zarte Geschlecht für so blöd zu halten, dass es sich vom ach so prächtigen Rad, das der sonst gar nicht so elegant anmutende Pfau schlage, visuell schon beeindrucken lasse; zwar sei die Natur unschuldig, 
aber das Rad des Pfaus bestehe nur aus einer Anzahl Federn. So wie ein vorgängiger Freier ihr ein selbst verfasstes, kürzlich veröffentlichtes Buch als Referenz überreicht habe, worin man blättern könne: alles Papier.

Die so und ähnlich von Prinzessin B. verspotteten und durchwegs nicht in Betracht gezogenen Freier beschwerten sich schliesslich bitter beim König, der die Tochter erneut zur Audienz vorlud, um ihr erstens mitzuteilen, der von ihr vor einigen Wochen unvernünftig und trotzig wie ein Kind in den Teich geworfene Ring sei trotz langer Suche nicht wiedergefunden worden. Zweitens: Ihr widerborstiges, ja kratzbürstiges Verhalten, übers Ganze gesehen, beweise ihm, immerhin ihrem Vater, nur, dass sie sich eigentlich nach einem Gatten sehne, ob sie sich wegen solcher Sehnsüchte selbst hasse oder nicht. Ihr Ärger über alles und jedes entspreche in diesem Fall doch nur dem ganz gewöhnlichen Frust über ihren weiterhin dank Abweisung aller Interessenten unerfüllt bleibenden ureigenen Wunsch nach einem Partner. So stehe sie zu seiner Besorgnis sich selbst im Weg und handle nicht nur den bedauernswerten, sich abmühenden und trotzdem nur gedemütigten sowie mit Pfauen verglichenen, übrigens, schliesslich, ebenfalls sehnsüchtigen Freiern zuleide, sondern auch sich selbst und vergrössere ihren Ärger nur.
Schliesslich würden nicht nur die Freier wegen des nötigen sich in die Brust Werfens platzen, sondern vor allem sie selbst, nämlich seine Tochter, vor Ärger.

Eine solche Predigt hatte Prinzessin B. von ihrem schweigsamen Vater nicht erwartet. Ziemlich zerknirscht und zudem einigermassen erschrocken verbrachte sie mehrere Tage fast ausschliesslich im Schlosspark. Einmal entdeckte sie am Ufer des Teichs einen winzigen Frosch, der sich ungeschickt bewegte und wand. Sie hielt ihn für verstümmelt und hätte ihn beinahe schon zertreten, als sie feststellte, dass er um den Bauch einen Ring wie einen engen Gürtel trug. Möglicherweise hatte sich das Tier, bereits als Kaulquappe, im Ring verfangen und war diesen nicht mehr losgeworden. Es gelang Prinzessin B., das behinderte Fröschlein in die Hand zu fangen und den Ring vom zappelnden kleinen Tierleib abzustreifen. Mein kleiner Frosch, sagte die junge Dame, es geht dir wie mir selbst, auch mir könnte es eng um die Brust werden, und eng um den Hals ist mir bereits. Da siehst du die Königstochter, die sich von ihrem Vater immer noch die Leviten lesen lassen muss, die alles hat und, so jung sie ist, nur den ganzen Tag schluckt und seufzt, als wöge ihr Herz eine Tonne! Sie gab dem kleinen Tier einen flüchtigen Kuss auf den Rükken und liess es ins Wasser gleiten. 